DESY 05-124

HU-EP-05/31

SFB/CPP-05-32

\title{
Non-perturbative quark mass renormalization in two-flavor QCD
}

\author{
$\bar{A}_{L P H A}$ \\ Michele Della Morte ${ }^{a}$, Roland Hoffmann ${ }^{a}$, Francesco Knechtli ${ }^{a}$, \\ Juri Rolf ${ }^{a}$, Rainer Sommer ${ }^{b}$, Ines Wetzorke ${ }^{c}$ and Ulli Wolff ${ }^{a}$ \\ ${ }^{a}$ Institut für Physik, Humboldt Universität, \\ Newtonstr. 15, 12489 Berlin, Germany \\ ${ }^{b}$ DESY, Platanenallee 6, 15738 Zeuthen, Germany \\ ${ }^{c}$ John von Neumann-Institut für Computing NIC, \\ Platanenallee 6, 15738 Zeuthen, Germany
}

\begin{abstract}
The running of renormalized quark masses is computed in lattice QCD with two flavors of massless $\mathrm{O}(a)$ improved Wilson quarks. The regularization and flavor independent factor that relates running quark masses to the renormalization group invariant ones is evaluated in the Schrödinger Functional scheme. Using existing data for the scale $r_{0}$ and the pseudoscalar meson masses, we define a reference quark mass in QCD with two degenerate quark flavors. We then compute the renormalization group invariant reference quark mass at three different lattice spacings. Our estimate for the continuum value is converted to the strange quark mass with the help of chiral perturbation theory.
\end{abstract}




\section{Introduction}

Lattice QCD provides a definition of quark masses from first principles. For example given a hadronic input accessible to experiments, such as the $\mathrm{K}$-meson mass $m_{\mathrm{K}}$ and decay constant $F_{\mathrm{K}}$, it is possible to compute the strange quark mass on the lattice. A convenient quantity to consider is the renormalization group invariant (RGI) strange quark mass, which is independent of the renormalization scheme if the renormalization conditions are imposed at zero quark mass [1].

If one looks at the most recent lattice results for the strange quark mass, from simulations with two [2-4] and two plus one [5,6] dynamical quark-flavours, there is a large spread of values ranging from $68 \mathrm{MeV}$ to $132 \mathrm{MeV}$. There are several sources of systematic errors in these computations, such as the use of perturbative renormalization (except for [4], where non-perturbative renormalization is done in the RI-MOM scheme) and the values of the lattice spacing affordable nowadays.

To perform a completely controlled computation we start from the bare PCAC mass $m_{i}\left(g_{0}\right)$ for a given quark flavor $i$ and determine the RGI mass

$$
M_{i}=Z_{\mathrm{M}}\left(g_{0}\right) m_{i}\left(g_{0}\right) \text {. }
$$

The bare coupling $g_{0}$ is in a one-to-one relation to the lattice spacing and the continuum limit of $M_{i}$ exists (and should be taken). As an intermediate step we first define the running mass

$$
\bar{m}_{i}(\mu)=\frac{Z_{\mathrm{A}}\left(g_{0}\right)}{Z_{\mathrm{P}}\left(g_{0}, a \mu\right)} m_{i}\left(g_{0}\right),
$$

where $\bar{m}$ is non-perturbatively defined in the Schrödinger Functional renormalization scheme and hence is well-defined also for low energies $\mu$. This corresponds to splitting $Z_{\mathrm{M}}$ into two factors

$$
Z_{\mathrm{M}}\left(g_{0}\right)=\frac{M}{\bar{m}(\mu)} \times \frac{Z_{\mathrm{A}}\left(g_{0}\right)}{Z_{\mathrm{P}}\left(g_{0}, a \mu\right)},
$$

which will be computed with full non-perturbative precision following the strategy of [7]. Note that all renormalization factors are flavor-independent and we hence omit the subscript $i$ in mass ratios. In the first factor of the above splitting the universal continuum limit is understood to have been taken. Its computation is the main objective of this paper. This result can then be used for any action and only the second factor needs to be redetermined. This becomes tractable by making the universal factor available for $\mu$ in the range of hadronic energies of $\mathrm{O}(1-2 \mathrm{GeV})$. As for the running coupling [8] our method thus avoids the need to treat a multi-scale problem in a large volume in this step. 
First results on the $\mu$-dependence of $\bar{m}$ in the $N_{\mathrm{f}}=2$ theory have already appeared in Refs. [9-11]. Beyond a finalization of these results, we here compute the second factor - and hence $Z_{\mathrm{M}}$ - using non-perturbative improvement [12] and renormalization [13] of the axial current in the theory with two flavors of $\mathrm{O}(a)$ improved Wilson quarks and plaquette gauge action.

As an application, one wants to determine the light (up, down, strange) quark masses. ${ }^{1}$ For practical reasons this is at present not yet possible for us in the most straight-forward way by simulations at the physical parameters. We need a hierarchy of additional approximations to compute for instance the strange quark mass.

First of all our simulation algorithm is at the moment still restricted to pairs of degenerate flavours and we include one such pair $\left(N_{\mathrm{f}}=2\right)$. In the large volume simulations needed to determine $m_{i}\left(g_{0}\right)$ in Eq. (1.1) our masses can at present not be taken to values small enough for the up- and down-flavors, for instance by tuning the pseudoscalar states to the physical pion mass. Instead we shall determine the RGI quark mass $M_{\text {ref }}$ that is associated with a "Kaon" made from two degenerate flavours.

The analogous computation of $M_{\text {ref }}$ has been performed in the quenched $N_{\mathrm{f}}=0$ theory [17] with a result that agrees within errors with the new one. We therefore assume that at the present level of accuracy it also applies to a hypothetical QCD with three degenerate flavors. A relation between this model and real QCD is finally established by chiral perturbation theory $[18,19]$ supplemented with some knowledge [19-21] of the phenomenologically inaccessible [22] low energy constants. The conclusion of [19] is that the ratio of quark masses is close to the one given at lowest order in chiral perturbation theory. Given this "fact" but keeping in mind that it should be scrutinized in future lattice QCD computations - it is then sufficient to compute any one quark mass from lattice QCD. In particular we can connect our $M_{\text {ref }}$ with the strange mass $M_{\mathrm{s}}$. The result that corresponds to the Gell-Mann-Oakes-Renner formula [23] is $M_{\mathrm{s}}=48 / 25 M_{\text {ref }}$.

Finally, the conversion of the RGI mass to the conventionally cited $\overline{\mathrm{MS}}$ mass at $2 \mathrm{GeV}$ renormalization scale is of course based on perturbation theory, which does however look very well behaved, see Table 6 .

\footnotetext{
${ }^{1}$ Later, when lattice spacings are small enough, the charm quark mass can be determined $[14,15]$. Also the beauty quark mass computed in HQET through the strategy of [16], is based on Eq. (1.1) in QCD.
} 


\section{The renormalization scheme}

QCD is a theory which has as free parameters the bare gauge coupling $g_{0}$ and the bare quark masses $m_{i}, i=1, \ldots, N_{\mathrm{f}}$. The hadronic scales like $F_{\mathrm{K}}$ or $m_{\mathrm{K}}$ are connected to the perturbative high energy regime of QCD via the running of renormalized couplings

$$
\bar{g}^{2}(\mu)=Z_{g} g_{0}^{2}, \quad \bar{m}_{i}(\mu)=Z_{m} m_{i}
$$

where $\mu$ is the renormalization scale. In the following we assume that the renormalization conditions are imposed at zero quark mass (mass-independent schemes) [1]. Introducing a regularization prescription, e.g. a lattice spacing $a$, the renormalization factors are functions of $g_{0}$ and $a \mu$

$$
Z_{g}=Z_{g}\left(g_{0}, a \mu\right), \quad Z_{m}=Z_{m}\left(g_{0}, a \mu\right) .
$$

In renormalized quantities the regulator can be removed, e.g. the continuum limit $a \rightarrow 0$ can be taken, yielding a finite result. The advantage of mass-independent renormalization schemes is that in all such schemes the ratios of renormalized quark masses for different flavors are scale and scheme independent constants $[19,24]$.

The running of the renormalized couplings is described by the renormalization group equations (RGE)

$$
\mu \frac{\mathrm{d} \bar{g}}{\mathrm{~d} \mu}=\beta(\bar{g}), \quad \mu \frac{\mathrm{d} \bar{m}_{i}}{\mathrm{~d} \mu}=\tau(\bar{g}) \bar{m}_{i} .
$$

The $\beta$ and $\tau$ functions are non-perturbatively defined if this is true for $\bar{g}$ and $\bar{m}_{i}$. Their perturbative expansions are

$$
\begin{aligned}
& \beta(\bar{g}) \underset{\bar{g} \rightarrow 0}{\sim}-\bar{g}^{3}\left\{b_{0}+b_{1} \bar{g}^{2}+b_{2} \bar{g}^{4}+\ldots\right\}, \\
& \tau(\bar{g}) \underset{\bar{g} \rightarrow 0}{\sim}-\bar{g}^{2}\left\{d_{0}+d_{1} \bar{g}^{2}+\ldots\right\} .
\end{aligned}
$$

The coefficients

$$
b_{0}=\frac{1}{(4 \pi)^{2}}\left(11-\frac{2}{3} N_{\mathrm{f}}\right), \quad b_{1}=\frac{1}{(4 \pi)^{4}}\left(102-\frac{38}{3} N_{\mathrm{f}}\right), \quad d_{0}=\frac{8}{(4 \pi)^{2}},
$$

are scheme independent. A physical quantity $P$ is a quantity for which the total dependence on the renormalization scale $\mu$ vanishes, i.e. it is a renormalization group invariant (RGI)

$$
\mu \frac{\mathrm{d}}{\mathrm{d} \mu} P\left(\mu, \bar{g},\left\{\bar{m}_{i}\right\}\right)=0
$$


Examples are the $\Lambda$-parameter and the RGI quark masses

$$
\begin{aligned}
\Lambda & =\mu\left(b_{0} \bar{g}^{2}\right)^{-b_{1} / 2 b_{0}^{2}} \mathrm{e}^{-1 /\left(2 b_{0} \bar{g}^{2}\right)} \exp \left\{-\int_{0}^{\bar{g}} \mathrm{~d} x\left[\frac{1}{\beta(x)}+\frac{1}{b_{0} x^{3}}-\frac{b_{1}}{b_{0}^{2} x}\right]\right\}, \\
M_{i} & =\bar{m}_{i}\left(2 b_{0} \bar{g}^{2}\right)^{-d_{0} / 2 b_{0}} \exp \left\{-\int_{0}^{\bar{g}} \mathrm{~d} x\left[\frac{\tau(x)}{\beta(x)}-\frac{d_{0}}{b_{0} x}\right]\right\}
\end{aligned}
$$

where $\bar{g}=\bar{g}(\mu)$ and $\bar{m}_{i}=\bar{m}_{i}(\mu)$. The $\Lambda$-parameter and the RGI quark masses are defined independent of perturbation theory and their connections between different mass independent renormalization schemes can be given in a simple and exact way [24]. In particular the RGI quark masses $M_{i}$ are scheme independent. Any physical quantity $P$ can be considered to be a function of $\Lambda$ and $M_{i}$, i.e. there exist a function $\hat{P}$ such that [24]

$$
P\left(\mu, \bar{g},\left\{\bar{m}_{i}\right\}\right)=\hat{P}\left(\Lambda,\left\{M_{i}\right\}\right)
$$

For this reason $\Lambda$ and $M_{i}$ seem preferable as the fundamental parameters of QCD.

\subsection{Quark masses in the Schrödinger Functional}

In QCD a renormalized mass is defined through the partially conserved axial current (PCAC) relation, which involves the renormalized axial current $\left(A_{\mathrm{R}}\right)_{\mu}(x)$ and the renormalized pseudoscalar density $P_{\mathrm{R}}(x)$

$$
\begin{aligned}
\partial_{\mu}\left(A_{\mathrm{R}}\right)_{\mu} & =\left(\bar{m}_{i}+\bar{m}_{j}\right) P_{\mathrm{R}}, \\
\left(A_{\mathrm{R}}\right)_{\mu}(x) & =Z_{\mathrm{A}} \bar{\psi}_{i}(x) \gamma_{\mu} \gamma_{5} \psi_{j}(x), \\
P_{\mathrm{R}}(x) & =Z_{\mathrm{P}} \bar{\psi}_{i}(x) \gamma_{5} \psi_{j}(x) .
\end{aligned}
$$

The renormalization constant $Z_{\mathrm{A}}$ can be calculated non-perturbatively, using chiral Ward identities and does not depend on the renormalization scale.

The renormalization constant $Z_{\mathrm{P}}$ can be conveniently determined in the Schrödinger Functional (SF) renormalization scheme [25,26]. There QCD is formulated in a finite box of spatial size $L$ and temporal extent $T$. The fields are subject to Dirichlet boundary conditions in time, which provide an infrared cutoff to the frequency spectrum of quarks and gluons. This allows to perform simulations at zero quark mass and thus to use the $\mathrm{SF}$ as a mass-independent renormalization scheme. Our renormalization scheme is further specified by setting $T=L$ (see below). The renormalization conditions are then naturally imposed at the scale $\mu=1 / L$.

The presence of boundary values for the fields in the SF formulation of a field theory requires in general additional (compared to the case without boundaries) 


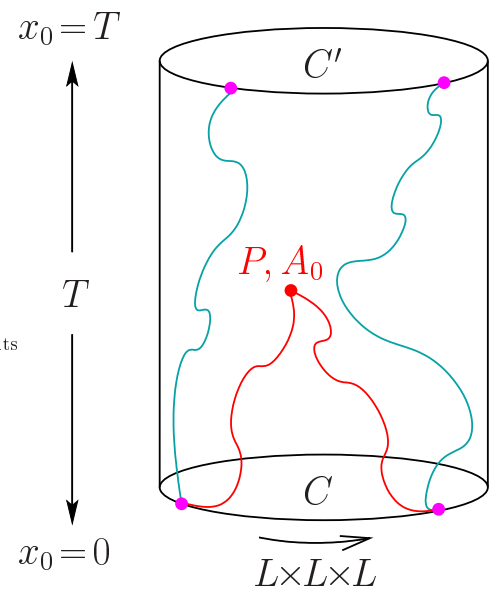

Figure 1: The Schrödinger Functional.

renormalization $[27,28]$. The renormalizability of QCD with SF boundaries has been studied in Refs. [29-32] where it was shown that no additional counterterms are needed except for one boundary term which amounts to a rescaling of the boundary values of the fermion fields by a logarithmically divergent factor.

The renormalization condition for $Z_{\mathrm{P}}$ that we employ is discussed in Refs. $[7,24,25]$. It uses a correlation function $f_{\mathrm{P}}\left(x_{0}\right)$, which is a matrix element of the pseudoscalar density inserted at time distance $x_{0}$ from a pseudoscalar boundary state, the other boundary state having vacuum quantum numbers. To cancel the multiplicative renormalization of the boundary quark fields the boundary-toboundary correlation function $f_{1}$ is used. The renormalization constant $Z_{\mathrm{P}}$ is then defined through

$$
Z_{\mathrm{P}}=\frac{\sqrt{3 f_{1}}}{f_{\mathrm{P}}(L / 2)} \quad \text { at } \quad m_{i}=0, i=1, \ldots, N_{\mathrm{f}} .
$$

The correlation functions are schematically represented in Fig. 1. They are computed at zero quark masses $m_{i}$. The definition Eq. (2.14) is such that $Z_{\mathrm{P}}=1$ at tree level of perturbation theory. The renormalization condition for $Z_{\mathrm{P}}$ is further specified by setting

$$
T=L, \quad C=C^{\prime}=0, \quad \theta=0.5
$$

where $C, C^{\prime}$ are the boundary gauge fields in the Lie algebra at $x_{0}=0$ and $x_{0}=T$ and $\theta$ the parameter controlling the spatial boundary conditions of the fermion fields. For more details of the calculation we refer to $[7,24]$.

A rigorous definition of the renormalized mass can be given in the lattice regularization of QCD. In our case we work with $N_{\mathrm{f}}=2$ mass-degenerate flavors 
of $\mathrm{O}(a)$ improved $[33,34]$ Wilson fermions. The massless theory is defined in the bare parameter space along the line $\kappa=\kappa_{c}\left(g_{0}\right)$ where the PCAC mass

$$
m\left(g_{0}, \kappa\right)=\left.\frac{\frac{1}{2}\left(\partial_{0}^{*}+\partial_{0}\right) f_{\mathrm{A}}\left(x_{0}\right)+c_{\mathrm{A}} a \partial_{0}^{*} \partial_{0} f_{\mathrm{P}}\left(x_{0}\right)}{2 f_{\mathrm{P}}\left(x_{0}\right)}\right|_{x_{0}=T / 2}
$$

vanishes. Here, $\partial_{0}$ and $\partial_{0}^{*}$ are the forward and backward lattice derivatives, respectively. The $\mathrm{O}(a)$ improvement coefficient $c_{\mathrm{A}}$ of the axial current has been computed non-perturbatively in Ref. [12]. The correlation function $f_{\mathrm{A}}\left(x_{0}\right)$ of the axial current $A_{0}$ is defined analogously to $f_{\mathrm{P}}\left(x_{0}\right)$. The renormalized PCAC mass at the scale $\mu=1 / L$ can then be written as

$$
\bar{m}(\mu)=\left.\lim _{a \rightarrow 0} Z_{m}\left(g_{0}, a \mu\right) m\left(g_{0}, \kappa\right)\right|_{u=\bar{g}^{2}(L)}, \quad Z_{m}\left(g_{0}, a \mu\right)=\frac{Z_{\mathrm{A}}\left(g_{0}\right)}{Z_{\mathrm{P}}\left(g_{0}, L / a\right)},
$$

where the renormalized gauge coupling $\bar{g}^{2}(L)$ [8] is kept fixed. Since the coupling runs with the box size $L$, keeping $\bar{g}^{2}$ fixed means keeping the renormalization scale $\mu$ fixed. The renormalization factor $Z_{\mathrm{A}}$ of the axial current has been determined non-perturbatively in Ref. [13]. The precise definition of $Z_{\mathrm{P}}$ (differing at order $a^{2}$ from Eq. (2.14)) is

$$
Z_{\mathrm{P}}\left(g_{0}, L / a\right)=c \frac{\sqrt{3 f_{1}}}{f_{\mathrm{P}}(L / 2)} \quad \text { at } \quad \kappa=\kappa_{c},
$$

where the factor $c(L / a)$ is chosen such that $Z_{P}(0, L / a)=1$ and can be found in $[7]$.

In order that the continuum limit is reached with cutoff effects strictly proportional to $a^{2}$, the $O(a)$ improvement factor $\left(1+\left(b_{\mathrm{A}}-b_{\mathrm{P}}\right) a m_{\mathrm{q}}\right)$ should be included in Eq. (2.17) [33]. Here $a m_{\mathrm{q}}=\left(1 / \kappa-1 / \kappa_{c}\right) / 2$ is the bare subtracted quark mass. Results in perturbation theory [35] and in the quenched approximation [36] show that the difference of improvement coefficients $b_{\mathrm{A}}-b_{\mathrm{P}}$ is small. The quark masses in our simulations will also be relatively small and we expect corrections due to $b_{\mathrm{A}}-b_{\mathrm{P}}$ at the per mille level, which we neglect.

\section{The running of the mass in the $\mathrm{SF}$-scheme}

The running of the renormalized quark mass $\bar{m}(\mu)$ in the SF scheme as specified in Section 2.1 with $N_{\mathrm{f}}=2$ mass-degenerate flavors can be computed on the lattice from the step scaling function of the renormalization factor $Z_{\mathrm{P}}$ extrapolated to the continuum

$$
\sigma_{\mathrm{P}}(u)=\lim _{a \rightarrow 0} \Sigma_{\mathrm{P}}(u, a / L), \quad \Sigma_{\mathrm{P}}(u, a / L)=\left.\frac{Z_{\mathrm{P}}\left(g_{0}, 2 L / a\right)}{Z_{\mathrm{P}}\left(g_{0}, L / a\right)}\right|_{u=\bar{g}^{2}(L)} .
$$




\begin{tabular}{cll}
\hline & \multicolumn{1}{c}{$\sigma_{\mathrm{P}}(u)$} & $\chi^{2} / n_{\mathrm{df}}$ \\
\hline 0.9793 & $0.9654(9)(11)$ & 2.16 \\
1.1814 & $0.9527(11)(6)$ & 0.47 \\
1.5031 & $0.9413(16)(2)$ & 0.01 \\
2.0142 & $0.9174(16)(24)$ & 3.58 \\
2.4792 & $0.8871(23)(18)$ & 0.54 \\
3.3340 & $0.8384(35)(12)$ & 0.20 \\
\hline
\end{tabular}

Table 1: Continuum extrapolations of $\Sigma_{\mathrm{P}}$ fitting the $L / a=8$ and $L / a=12$ data to a constant. The first error is statistical. The second error is the difference between the fit and the $L / a=8$ results and will be added linearly as a systematic error.

From Eq. (2.17) it follows immediately that

$$
\sigma_{\mathrm{P}}(u)=\frac{\bar{m}(\mu)}{\bar{m}(\mu / 2)} \text { for } \mu=1 / L
$$

i.e. the step scaling function $\sigma_{\mathrm{P}}(u)$ describes the running of the renormalized quark mass. We computed $\Sigma_{\mathrm{P}}(u, a / L)$ at six values of the renormalized coupling $u$ corresponding approximately to a range of box sizes of the order $L=10^{-2} \mathrm{fm} \ldots 1 \mathrm{fm}$ (or equivalently $\mu$ of the order $100 \mathrm{GeV} \ldots 1 \mathrm{GeV}$ ). At each value of $u$ we simulated three lattice resolutions $L / a=6,8,12$ and the results for $Z_{\mathrm{P}}$ and $\Sigma_{\mathrm{P}}$ are summarized in Table 7 in Appendix A. For the extrapolation to the continuum, we fitted to a constant the two values of $\Sigma_{\mathrm{P}}$ on the finer lattices, separately for each coupling $u$. We then added linearly the difference between the fit and the $L / a=8$ result as a systematic error. The continuum estimates can be seen in Fig. 2. Our data do not show any significant dependence on the lattice spacing, as we could verify by trying different extrapolations (quadratic, linear in $a$ ). This statement is based on the statistical accuracy that we could achieve. We remark that also in the quenched approximation the cutoff effects were found to be small [7] and there $\Sigma_{P}$ was computed at an even finer lattice resolution $L / a=16$. The continuum values of $\sigma_{\mathrm{P}}(u)$ are summarized in Table 1. In the last column we list the $\chi^{2}$ divided by the number of degrees of freedom $n_{\mathrm{df}}$ of the fit. Their average is close to the expected value of one.

In perturbation theory the step scaling function $\sigma_{\mathrm{P}}(u)$ has an expansion $\sigma_{\mathrm{P}}(u)=1-\ln (2) d_{0} u+\mathrm{O}\left(u^{2}\right)$. In Fig. 3 our non-perturbative data for $\sigma_{\mathrm{P}}(u)$ are conveniently plotted for comparison with perturbation theory. We relate $\sigma_{\mathrm{P}}(u)$ to 


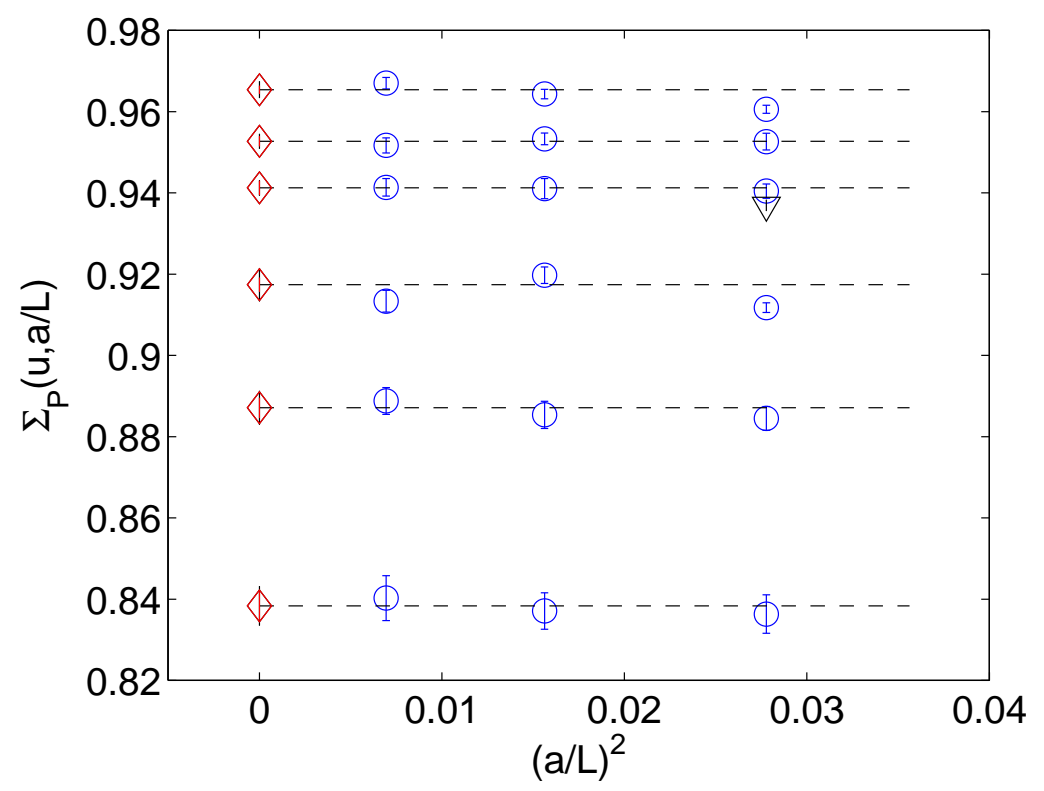

Figure 2: Continuum extrapolations of $\Sigma_{\mathrm{P}}$. The $L / a=6$ data have been excluded from the fits. For the third smallest coupling, the two points at $L / a=6$ refer to 1 -loop and 2-loop values for the boundary improvement coefficient $c_{t}[29,37]$.

$\tau$ and $\beta$ using Eq. (2.9)

$$
\sigma_{\mathrm{P}}(u)=\left(\frac{u}{\sigma(u)}\right)^{d_{0} /\left(2 b_{0}\right)} \exp \left\{-\int_{\sqrt{u}}^{\sqrt{\sigma(u)}} \mathrm{d} x\left[\frac{\tau(x)}{\beta(x)}-\frac{d_{0}}{b_{0} x}\right]\right\}
$$

where $\sigma(u)$ is the step scaling function of the coupling and is determined by [8]

$$
-2 \ln (2)=\int_{u}^{\sigma(u)} \mathrm{d} x \frac{1}{\sqrt{x} \beta(\sqrt{x})} .
$$

Using for the $\tau$-function the 2-loop expression with [24]

$$
d_{1}=d_{0}\left(0.0271+0.0105 N_{\mathrm{f}}\right)
$$

and for the $\beta$-function the 3 -loop expression with (see [8])

$$
b_{2}=\left(0.483-0.275 N_{\mathrm{f}}+0.0361 N_{\mathrm{f}}^{2}-0.00175 N_{\mathrm{f}}^{3}\right) /(4 \pi)^{3}
$$

we get from Eq. (3.3) and Eq. (3.4) the perturbative curve shown in Fig. 3. Our non-perturbative data do not show any significant deviation from the perturbative estimates. 


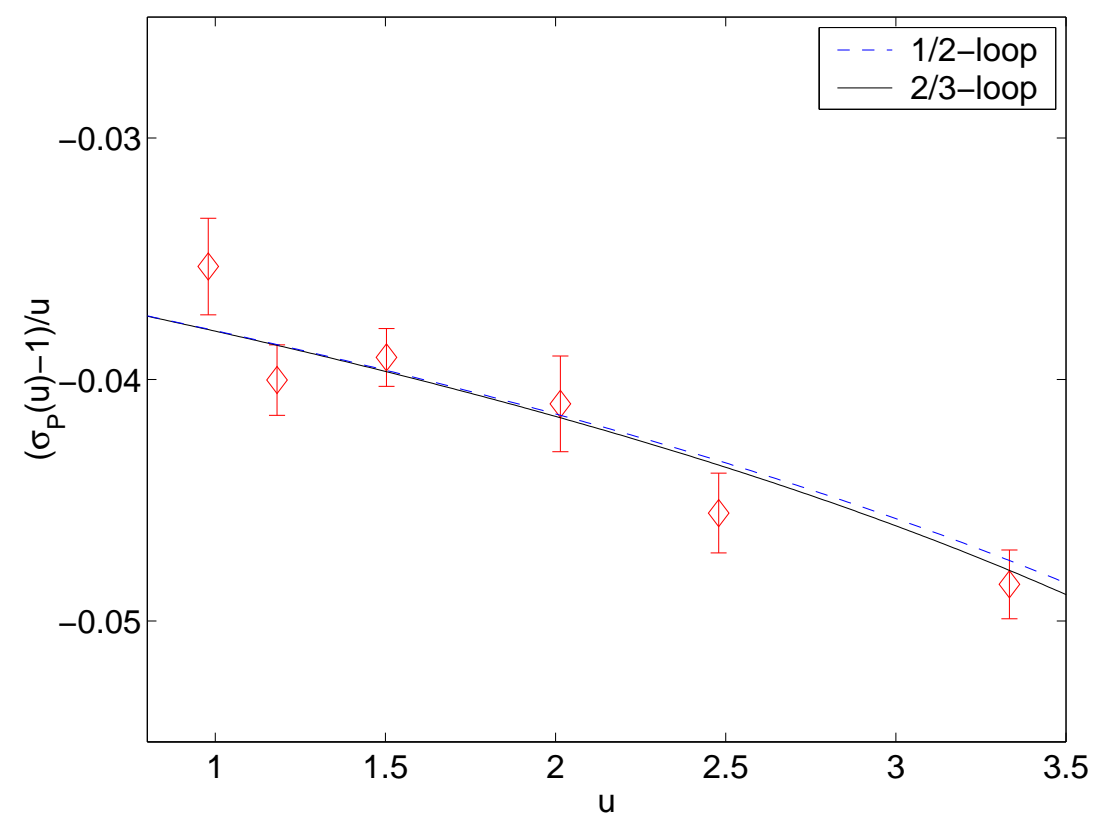

Figure 3: Comparison of the non-perturbative data for the step scaling function $\sigma_{\mathrm{P}}(u)$ with perturbation theory. 2/3-loop refers to the 2 -loop $\tau$-function and 3 -loop $\beta$-function, analogously $1 / 2-$ loop.

We now take the continuum values for $\sigma_{\mathrm{P}}(u)$ in Table 1 (with added statistical and systematic errors) and for the step scaling function of the coupling $\sigma(u)$ the results from fits to constants in Table 4 of Ref. [8]. We solve the following joint recursion to evolve coupling and mass from a low energy scale $1 / L_{\max }$ defined by

$$
u_{0}=\bar{g}^{2}\left(L_{\max }\right)=4.61
$$

to the higher scales $1 / L_{k}, k=0,1, \ldots, 8$ (with $L_{0} \equiv L_{\max }$ )

$$
\begin{aligned}
& \left\{\begin{array}{l}
u_{0}=\bar{g}^{2}\left(L_{\max }\right)=4.61 \\
\sigma\left(u_{k+1}\right)=u_{k}
\end{array} \Rightarrow u_{k}=\bar{g}^{2}\left(L_{k}\right), \quad L_{k}=2^{-k} L_{\max },\right. \\
& \left\{\begin{array}{l}
w_{0}=1 \\
w_{k}=\left[\prod_{i=1}^{k} \sigma_{\mathrm{P}}\left(u_{i}\right)\right]^{-1}
\end{array}\right.
\end{aligned}
$$

We interpolate the values of $\sigma(u)$ and $\sigma_{\mathrm{P}}(u)$ through a polynomial ansatz

$$
\begin{aligned}
\sigma(u) & =u+s_{0} u^{2}+s_{1} u^{3}+s_{2} u^{4}+s_{3} u^{5}+s_{4} u^{6}, \\
\sigma_{\mathrm{P}}(u) & =1+p_{0} u+p_{1} u^{2}+p_{2} u^{3},
\end{aligned}
$$

where the coefficients $s_{0}, s_{1}$ [8] and $p_{0}=-\ln (2) d_{0}$ are fixed to their perturbative values. The coefficients $s_{2}, s_{3}, s_{4}$ and $p_{1}, p_{2}$ are here fit parameters. The errors of the recursion coefficients are computed by error propagation. 


\begin{tabular}{|c|c|c|c|}
\hline$k$ & $u_{k}$ & $\begin{array}{c}M / \bar{m}\left(1 / L_{\max }\right) \\
2 / 3-\text { loop }\end{array}$ & $\begin{array}{c}M / \bar{m}\left(1 / L_{\max }\right) \\
1 / 2 \text {-loop }\end{array}$ \\
\hline 0 & 4.61 & 1.274 & 1.267 \\
\hline 1 & $3.032(16)$ & $1.296(6)$ & 1.292 \\
\hline 2 & $2.341(21)$ & $1.295(10)$ & 1.292 \\
\hline 3 & $1.918(20)$ & $1.294(13)$ & 1.292 \\
\hline 4 & $1.628(16)$ & $1.294(14)$ & 1.292 \\
\hline 5 & $1.414(14)$ & $1.295(15)$ & 1.293 \\
\hline 6 & $1.251(12)$ & $1.297(16)$ & 1.295 \\
\hline 7 & $1.121(10)$ & $1.298(17)$ & 1.297 \\
\hline 8 & $1.017(10)$ & $1.299(17)$ & 1.298 \\
\hline
\end{tabular}

Table 2: Values for $M / \bar{m}\left(1 / L_{\max }\right)$ from Eq. (3.12).

Using the coefficients $w_{k}$ in Eq. (3.9) we compute

$$
\frac{M}{\bar{m}\left(1 / L_{\max }\right)}=w_{k}^{-1} \frac{M}{\bar{m}\left(1 / L_{k}\right)},
$$

where the factor $M / \bar{m}\left(1 / L_{k}\right)$ is calculated from Eq. (2.9) with $\bar{g}^{2}=u_{k}$ by employing the perturbative expressions for the $\tau$ - and $\beta$-functions at 2 - respectively 3 -loop order. The results are shown in Table 2 . They have a remarkable stability in the coupling ${ }^{2} u_{k}$ and we take $k=6$ as our result

$$
\frac{M}{\bar{m}(\mu)}=1.297(16) \quad \text { at } \quad \mu=1 / L_{\max }
$$

We emphasize that from Eq. (2.9) it is evident that $\sigma_{\mathrm{P}}(u)$ and $M / \bar{m}(\mu)$ are flavor independent. Moreover, since the continuum limit has been taken any regularization dependence has been removed from the result Eq. (3.13).

Finally, in Fig. 4 we plot the non-perturbative running of the renormalized mass. For $\mu / \Lambda=1 /\left(L_{k} \Lambda\right), k=0,1, \ldots, 8$, we plot the points $\bar{m}\left(1 / L_{k}\right) / M$ obtained from Eq. (3.12) using the result Eq. (3.13). The physical scale $\Lambda$ is here implicitly determined through $\ln \left(\Lambda L_{\max }\right)=-1.298(58)$ obtained from the recursion Eq. (3.8). In the plot we neglect the overall uncertainties of $\bar{m}\left(1 / L_{\max }\right) / M$ and $\ln \left(\Lambda L_{\max }\right)$, since they would simply change the scales on the plot axes. The

\footnotetext{
${ }^{2}$ The deviation in the case $k=0$ is due to the difference between the perturbative and the non-perturbative values of $\sigma(u)$ at large $u$ (see [8]).
} 


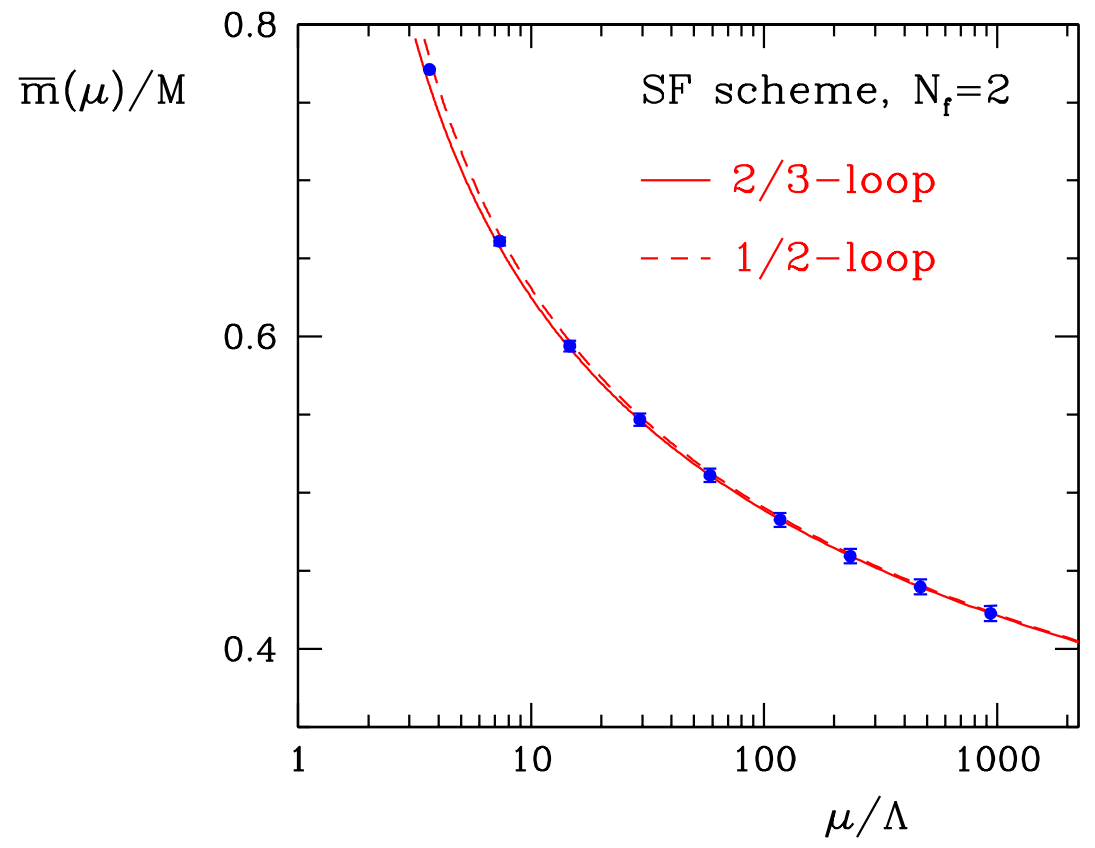

Figure 4: The non-perturbative running of $\bar{m}$. 2/3-loop refers to the 2 -loop $\tau$-function and 3 -loop $\beta$-function, analogously $1 / 2-$ loop.

errors of the points in Fig. 4 come from the coefficients $w_{k}$. Together with the non-perturbative points we show the perturbative curves that are obtained from Eq. (2.8) and Eq. (2.9) by using the perturbative expressions for the $\tau$ - and $\beta$-functions at $1-, 2$ - respectively $2-, 3$-loop order. The non-perturbative and perturbative running are very close down to the smallest energies that were accessible in our simulations. We remark that this statement explicitly refers to the special SF renormalization scheme considered here.

\section{Estimate of the strange quark mass}

\subsection{Complete $Z_{\mathrm{M}}$ for the improved Wilson discretization}

We now derive the second factor in Eq. (1.3) for a few values of the lattice spacing or respectively the bare coupling. As emphasized before, this contribution is non-universal and in the form given it will be valid only for our action of non- 


\begin{tabular}{cccll}
\hline$\beta$ & $\kappa$ & $L / a$ & \multicolumn{1}{c}{$\bar{g}^{2}$} & \multicolumn{1}{c}{$Z_{\mathrm{P}}$} \\
\hline 5.20 & 0.13600 & 4 & $3.65(3)$ & $0.55188(51)$ \\
5.20 & 0.13600 & 6 & $4.61(4)$ & $0.47876(47)$ \\
5.29 & 0.13641 & 4 & $3.394(17)$ & $0.57643(50)$ \\
5.29 & 0.13641 & 6 & $4.279(37)$ & $0.50739(58)$ \\
5.29 & 0.13641 & 8 & $5.65(9)$ & $0.45799(75)$ \\
5.40 & 0.13669 & 4 & $3.188(24)$ & $0.60269(65)$ \\
5.40 & 0.13669 & 6 & $3.861(34)$ & $0.53681(54)$ \\
5.40 & 0.13669 & 8 & $4.747(63)$ & $0.49116(66)$ \\
\hline
\end{tabular}

Table 3: Results for $Z_{\mathrm{P}}, c_{t}$ set to 2-loop value. The values of $\bar{g}^{2}$ are from [8]. The hopping parameters $\kappa$ are set to the critical ones $\left(\kappa_{c}\right)$ of [4].

perturbatively improved Wilson fermions with plaquette gauge action and $c_{\mathrm{sw}}$ as specified in [34]. For $Z_{\mathrm{A}}$ and $c_{\mathrm{A}}$ we employ the values recently given and parameterized in [13] and [12]. It remains to compute $Z_{\mathrm{P}}\left(g_{0}, L_{\max } / a\right)$ for the desired values of the bare coupling $g_{0}$, here given by $\beta=5.2,5.29,5.4$. The scale $L_{\max }$ is fixed by $\bar{g}^{2}\left(L_{\max }\right)=4.61$, where the universal factor of $Z_{\mathrm{M}}$ is now known, Eq. (3.13). Our basis are the simulation results summarized in Table 3.

While the simulation at the largest bare coupling is exactly at the target value for $\bar{g}^{2}$, the two other series of simulations require a slight interpolation. This has been done using a fit ansatz motivated by Eq. (2.9)

$$
\ln \left(Z_{\mathrm{P}}\right)=c_{1}+c_{2} \ln \left(\bar{g}^{2}\right)
$$

to interpolate $Z_{\mathrm{P}}$ between two values of $\bar{g}^{2}$ straddling 4.61. The fit takes into account the (independent) errors of both $Z_{\mathrm{P}}$ and $\bar{g}^{2}$. The fit error is then augmented by the difference between the fit result from Eq. (4.1) and the result from a simple two point linear interpolation in $\bar{g}^{2}$. The values of the coefficient $c_{2}$ in the fit Eq. (4.1) are found to be $-0.369(25)$ at $\beta=5.29$ and $-0.430(34)$ at $\beta=5.40$, which are not far from $-d_{0} /\left(2 b_{0}\right)=-0.4138$.

The resulting numbers for $Z_{\mathrm{P}}$ and $Z_{\mathrm{M}}$ are summarized in Table 4. The first error for $Z_{\mathrm{M}}$ comes from the error of the factor $Z_{\mathrm{A}} / Z_{\mathrm{P}}$. The second error is the $1.2 \%$ uncertainty in the universal factor $M / \bar{m}$ and should be added in quadrature to the quark mass error after the continuum limit has eventually been taken. 


\begin{tabular}{clc}
\hline$\beta$ & \multicolumn{1}{c}{$Z_{\mathrm{P}}$} & \multicolumn{1}{c}{$Z_{\mathrm{M}}$} \\
\hline 5.20 & $0.47876(47)$ & $1.935(33)(24)$ \\
5.29 & $0.4936(34)$ & $1.979(25)(24)$ \\
5.40 & $0.4974(33)$ & $2.001(29)(25)$ \\
\hline
\end{tabular}

Table 4: Results for $Z_{\mathrm{P}}$ and finally $Z_{\mathrm{M}}$ for three coupling values. The action to which it refers is detailed in the text.

\subsection{The reference quark mass}

As announced in the introduction we next compute the reference quark mass $M_{\text {ref }}$ producing a pseudoscalar with the mass of the Kaon in our simulated two-flavour theory. The hopping parameter $\kappa_{\text {ref }}$ is tuned to keep the pseudoscalar mass $m_{\mathrm{PS}}$ in the relation

$$
\left(r_{0}\left(\kappa_{c}\right) m_{\mathrm{PS}}\left(\kappa_{\mathrm{ref}}\right)\right)^{2}=\left(r_{0} m_{\mathrm{K}}\right)^{2}=1.5736
$$

where $r_{0}$ is the scale extracted from the static quark potential [38]. This value corresponds to the $\mathrm{K}$-meson mass $m_{\mathrm{K}}^{2}=(495 \mathrm{MeV})^{2}$ for $r_{0}=0.5 \mathrm{fm}$ [17]. Using the data for $r_{0}(\kappa), m_{\mathrm{PS}}(\kappa)$ and $\kappa_{c}$ available in Ref. [4] and the extrapolations to the chiral limit $r_{0}\left(\kappa_{c}\right)$ of Ref. [8], we can safely perform a slight extrapolation to the value $\kappa=\kappa_{\text {ref }}$ defined in Eq. (4.2). We employ the following fit ansatz for $r_{0} m_{\mathrm{PS}}$ as a function of the bare subtracted quark mass $a m_{\mathrm{q}}$

$$
\frac{\left(r_{0}\left(\kappa_{c}\right) m_{\mathrm{PS}}(\kappa)\right)^{2}}{a m_{\mathrm{q}}}=e_{1}+e_{2} \cdot a m_{\mathrm{q}}
$$

with fit coefficients $e_{1}$ and $e_{2}$. The results of the fits are $\kappa_{\text {ref }}=0.135680(30)$ at $\beta=5.20, \kappa_{\text {ref }}=0.136018(27)$ at $\beta=5.29$ and $\kappa_{\text {ref }}=0.136293(25)$ at $\beta=5.40$.

The PCAC masses $a m_{\text {ref }}$ have been computed in simulations at $\kappa=\kappa_{\text {ref }}$ and the results are summarized in Table 5. In these simulations the parameter $\theta$ in Eq. (2.15) has been set to zero. Barring cutoff effects the PCAC mass is independent of the time $x_{0}$ at which the right hand side of Eq. (2.16) is evaluated. Therefore in Table 5 we average over a range $t_{1}: t_{2}$ of $x_{0}$ values around $x_{0}=$ $L / 2$, where the PCAC mass has a plateau. We keep $t_{1}-t_{2}$ roughly constant in physical units. We see a significant gain in statistical precision due to the averaging compared to taking only the time $x_{0}=L / 2$. The error analysis of derived observables like the (averaged) PCAC mass and $Z_{\mathrm{P}}$ has been done with the method of Ref. [39]. 


\begin{tabular}{ccccccc}
\hline$\beta$ & $\kappa_{\text {ref }}$ & $L / a$ & $t_{1} / a: t_{2} / a$ & $a m_{\text {ref }}$ & $M_{\text {ref }}[\mathrm{MeV}]$ & $\begin{array}{c}M_{\text {ref }}[\mathrm{MeV}] \\
\text { using } Z_{\mathrm{A}}^{\text {con }}\end{array}$ \\
\hline 5.20 & 0.135680 & 16 & $7: 9$ & $0.01410(30)$ & $58.7(3.2)$ & $69.5(3.7)$ \\
5.29 & 0.136018 & 16 & $6: 10$ & $0.01352(28)$ & $63.5(3.2)$ & $69.1(3.6)$ \\
5.40 & 0.136293 & 24 & $10: 14$ & $0.01300(18)$ & $72.0(2.7)$ & $76.2(2.7)$ \\
\hline
\end{tabular}

Table 5: Results for the PCAC masses and the RGI reference quark masses, the latter converted to $\mathrm{MeV}$ assuming $r_{0}=0.5 \mathrm{fm}$. Two definitions of $Z_{\mathrm{A}}$ are used [13].

The cutoff effects in $m_{\text {ref }}$ also depend on the volume. In fact, at a lattice spacing of $0.1 \mathrm{fm}$ this dependence is rather strong [40]. Thus one has to define the PCAC mass for a fixed volume in order to ensure that cutoff effects disappear smoothly as $\mathrm{O}\left(a^{2}\right)$. Furthermore it is preferable to choose $L$ relatively large. We choose $L \gtrsim 1.5 \mathrm{fm}$, where the volume dependence can be neglected. Indeed, we compared results from a simulation at $\beta=5.2, \kappa=0.1355, c_{\mathrm{sw}}=2.02$ on a $L / a=16$ lattice with those from the JLQCD collaboration ${ }^{3}$ obtained at the same parameters on a $20^{3} \times 48$ lattice [3]. We could check that at the level of $1.6 \%$ statistical precision in our simulation, the PCAC masses agree. We conclude that our volume is large enough at $\beta=5.2$ for the volume dependence to be negligible. Therefore at the higher $\beta$ values we choose approximately matched (or larger) physical volumes.

The second to last column of Table 5 shows our results for the reference RGI quark mass

$$
M_{\text {ref }}=Z_{\mathrm{M}} m_{\text {ref. }}
$$

We do see large cutoff effects. At $\beta=5.2$ similarly large cutoff effects have been observed in other quantities as well $[13,40,41]$. Moreover the lattice spacing in our simulations changes by $30 \%$ only when going from $\beta=5.2$ to $\beta=5.4$. We therefore do not attempt (and discourage) any elaborate continuum extrapolation based on this range of lattice spacings. Instead we use a conservative estimate of the continuum value of $M_{\text {ref }}$ by taking its value at our largest $\beta=5.4$ with the difference to the value at $\beta=5.2$ added as systematic error

$$
M_{\text {ref }}=72(3)(13) \mathrm{MeV} \text {. }
$$

This result makes it clear that at present the systematic error is dominating over the statistical one.

\footnotetext{
${ }^{3}$ We are grateful to Takashi Kaneko for providing us with data for this comparison.
} 
The last column of Table 5 shows the results for an alternative estimate of $M_{\text {ref }}$, which differs only by the use of the renormalization factor $Z_{\mathrm{A}}^{\text {con }}[13]$. We observe that the difference in $M_{\text {ref }}$ between the two choices for $Z_{\mathrm{A}}$ is compatible with an $a^{2}$-behavior. Actually, the data obtained with $Z_{\mathrm{A}}^{\text {con }}$ have a weaker $a^{-}$ dependence but this effect is essentially due to the change at the coarsest lattice spacing $(\beta=5.2)$.

\subsection{The strange quark mass}

To make contact with physics we now assume that Eq. (4.5) also holds within errors in a theory with three degenerate flavors, which can then be related to the strange quark in QCD by chiral perturbation theory. This assumption is supported by the fact that the value of $M_{\text {ref }}$ Eq. (4.5) is the same within errors as in the $N_{\mathrm{f}}=0$ theory [17].

At lowest order in chiral perturbation theory, disregarding the electromagnetic interaction, the formula $[18,23,42]$

$$
m_{\mathrm{K}}^{2}=\frac{1}{2}\left(m_{\mathrm{K}^{+}}^{2}+m_{\mathrm{K}^{0}}^{2}\right)=\left(\hat{M}+M_{\mathrm{s}}\right) B_{\mathrm{RGI}},
$$

where $\hat{M}=1 / 2\left(M_{\mathrm{u}}+M_{\mathrm{d}}\right)$, holds. Here $M_{\mathrm{u}}, M_{\mathrm{d}}, M_{\mathrm{s}}$ are the up, down, strange RGI quark masses and $B_{\text {RGI }}$ is a constant of the chiral Lagrangian. Eq. (4.6) implies for degenerate quarks of RGI mass $M_{\text {ref }}$ (defined according to Eq. (4.2))

$$
m_{\mathrm{K}}^{2}=2 M_{\mathrm{ref}} B_{\mathrm{RGI}} .
$$

Therefore the relation $M_{\text {ref }}=\left(\hat{M}+M_{\mathrm{s}}\right) / 2$ holds at lowest order in chiral perturbation theory and using $M_{\mathrm{s}} / \hat{M}=24.4(1.5)$ [19] gives

$$
M_{\mathrm{s}} \approx 48 / 25 M_{\text {ref }} .
$$

Corrections to Eq. (4.8) are expected to be small in chiral perturbation theory $[17,20,43,44]$ and anyway below the accuracy that we will reach here for our result. In addition in the quenched approximation it was found that the dependence of pseudoscalar masses $m_{\mathrm{PS}}$, at fixed average quark mass, on the difference of quark masses is rather small [17]. We therefore assume Eq. (4.8) to hold also with dynamical quarks.

At this point we are ready to give an estimate of the continuum value of the RGI strange quark mass

$$
M_{\mathrm{s}}=138(5)(26) \mathrm{MeV},
$$




\begin{tabular}{cccc}
\hline$\mu[\mathrm{GeV}]$ & \multicolumn{3}{c}{$\bar{m}^{\overline{\mathrm{MS}}}(\mu) / M$} \\
& 2-loop & $3-$ loop & 4 -loop \\
\hline & & & \\
1.0 & 0.7979 & 0.8364 & 0.8469 \\
2.0 & 0.6824 & 0.6984 & 0.7013 \\
4.0 & 0.6110 & 0.6197 & 0.6209 \\
8.0 & 0.5608 & 0.5662 & 0.5668 \\
90.0 & 0.4571 & 0.4588 & 0.4589 \\
\hline
\end{tabular}

Table 6: Factors to convert the renormalization group invariant mass into the $\overline{\mathrm{MS}}$ scheme at scale $\mu$, for $\Lambda_{\overline{\mathrm{MS}}}^{(2)}=245(32)[8]$ and $N_{\mathrm{f}}=2$.

by combining Eq. (4.8) with Eq. (4.5). Equivalently to the determination of $M_{\text {ref }}$, from Eq. (4.7) and Eq. (4.5) we get

$$
B_{\mathrm{RGI}}=1.70(38) \mathrm{GeV} .
$$

The renormalized strange quark mass in the $\overline{\mathrm{MS}}$ scheme at the renormalization scale $\mu$ is obtained by multiplying $M_{\mathrm{s}}$ with the conversion factor $\bar{m}^{\overline{\mathrm{MS}}}(\mu) / M$. The latter is computed perturbatively by numerical integration of Eq. (2.8) and Eq. (2.9) and is listed in Table 6 for different choices of $\mu$. The $n=2,3,4$-loop approximations of the $\beta$ and $\tau$ functions in the $\overline{\mathrm{MS}}$ scheme (for $N_{\mathrm{f}}=2$ ) are used. The coefficients at 4-loop have been computed in [45-48]. To set $\mu$ in physical units the result $\Lambda_{\overline{\mathrm{MS}}}^{(2)}=245(32)$ of Ref. [8] is used. The uncertainty in the $\Lambda$ parameter translates into a $2.8 \%$ uncertainty in $\bar{m}^{\overline{\mathrm{MS}}}(\mu) / M$ at $\mu=2 \mathrm{GeV}$ and $4.9 \%$ at $\mu=1 \mathrm{GeV}$ (with 4 -loop evolution). Taking this into account our estimate for the $\overline{\mathrm{MS}}$ strange quark mass is

$$
\bar{m}_{\mathrm{s}}^{\overline{\mathrm{MS}}}(\mu)=97(22) \mathrm{MeV} \text { at } \mu=2 \mathrm{GeV} .
$$

\section{Conclusions and outlook}

We have presented a fully non-perturbative renormalization of the quark mass in two flavor QCD, with renormalization conditions specified at zero quark mass in the Schrödinger Functional scheme. Simulations were performed with $\mathrm{O}(a)$ improved Wilson quarks. Our main results are the running of the quark mass in Fig. 4 and the factor $M / \bar{m}(\mu)$ relating the quark mass at a specified low energy scale $\mu$ with the RGI quark mass in the continuum limit, Eq. (3.13). 


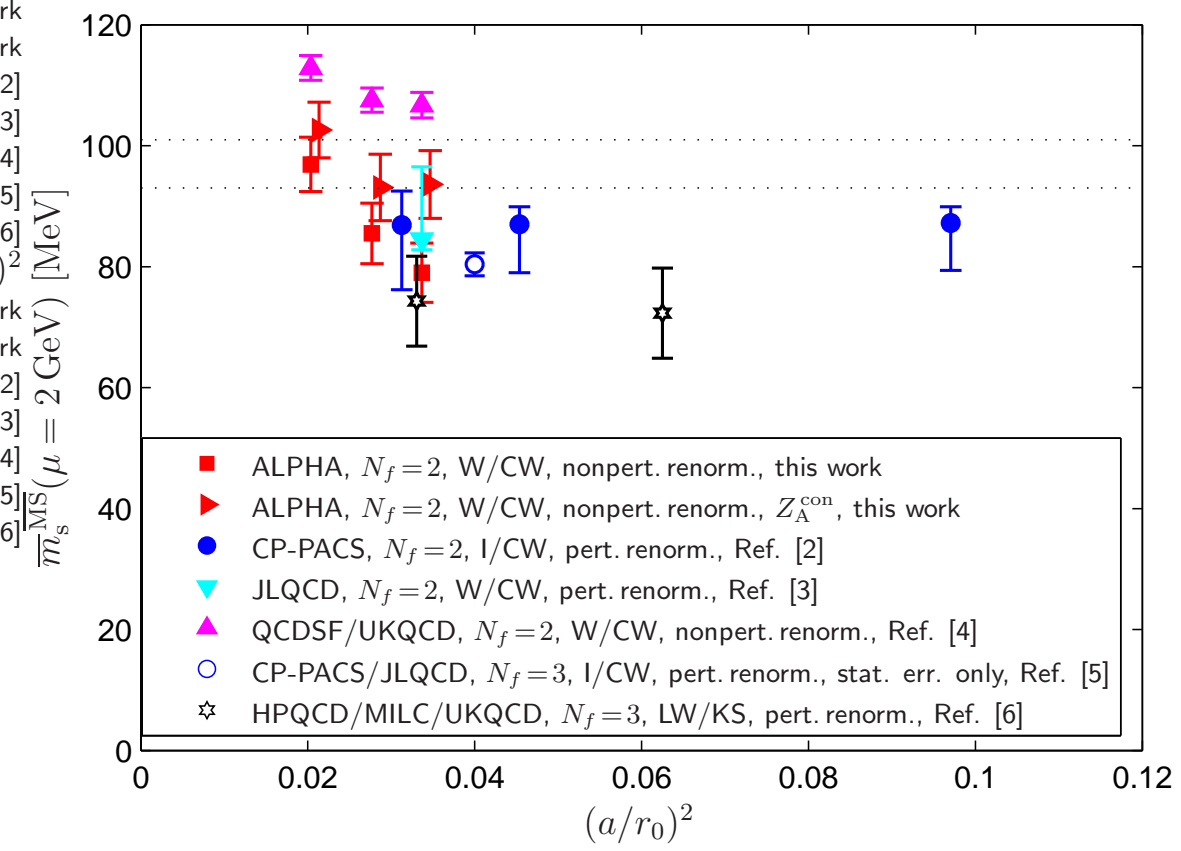

Figure 5: Summary of the strange quark mass data from lattice simulations. In the legend the discretizations used are indicated in the form gauge action/fermion action. The dictionary reads: W: Wilson gauge action; I: Iwasaki gauge action; LW: 1-loop tadpole improved Lüscher-Weisz gauge action; CW: Wilson-clover fermion action; KS: Asqtad staggered fermion action. The dotted lines represent the quenched result [17].

In order to obtain the renormalized strange quark mass in physical units an appropriate hadronic scheme has to be defined. By using existing data on the pseudoscalar masses and the scale $r_{0}$ from Ref. [4] we were able to determine the RGI reference quark mass $M_{\text {ref }}$ defined through Eq. (4.4) and Eq. (4.2), at three lattice spacings in the approximate range $0.092 \ldots 0.071 \mathrm{fm}$. Even in this small range we see distinct cutoff effects. At the largest lattice spacing large cutoff effects have been observed elsewhere $[13,40,41]$. These facts make it impossible to perform a systematic continuum extrapolation. Nevertheless we can give a conservative estimate of the continuum value in Eq. (4.5). We emphasize that the error in Eq. (4.5) is dominated by the systematic uncertainty of this step.

Using a number of additional reasonable assumptions we convert $M_{\text {ref }}$ to the strange quark mass in the $\overline{\mathrm{MS}}$ scheme Eq. (4.11). A definite clarification that all of these lead to negligible errors will require some future work.

In Fig. 5 we summarize the most recent results for the strange quark mass 
from lattice simulations with two [2-4] and two plus one [5,6] dynamical quarks. The physical $\mathrm{K}$-meson mass is always used as input although in some cases this is employed in a partially quenched setup where valence and sea quark masses differ, while we compute $M_{\text {ref }}$ and then use chiral perturbation theory to connect to the physical theory. In the legend we emphasize whether nonperturbative or perturbative renormalization has been used as well as the adopted discretizations. It appears that perturbative renormalization leads to rather small values for the strange quark mass. ${ }^{4}$ We plot the results of our present work using two renormalization conditions for $Z_{\mathrm{A}}$ that differ at $\mathrm{O}\left(a^{2}\right)$. The red triangles are obtained with $Z_{\mathrm{A}}^{\text {con }}[13]$ and are slightly displaced for clarity. This comparison gives a flavor of the quite large cutoff effects at $\beta=5.2$, our coarsest lattice spacing. The dotted lines in Fig. 5 mark the quenched result of Ref. [17]. Given the present status, illustrated in Fig. 5, it appears hard to claim a definite dependence of $m_{\mathrm{s}}$ on the number of dynamical fermions, even between $N_{\mathrm{f}}=0$ and $N_{\mathrm{f}}=2$.

Our present result for the renormalized strange quark mass should be improved by simulating at a finer lattice spacing. We are confident that this will be possible in the near future. There are promising improvements in algorithms [49-51], and a new machine apeNEXT is becoming available to us [52,53]. Moreover the determination of the lattice spacing can be improved by, for example, computing the renormalized strange quark mass in units of the $\mathrm{K}$-meson decay constant $F_{\mathrm{K}}$. There are first indications that the SF is an efficient setup for this low-energy computation (see also [54]).

Acknowledgement. We are grateful to Gerrit Schierholz for communicating results of Ref. [4]. We further thank NIC/DESY for allocating computing resources on the APEmille machine to this project and the APE collaboration and the staff of the computer center at DESY, Zeuthen for their support.

The computation of the renormalized quark mass is part of project B2 of the SFB Transregio 9 "Computational Particle Physics" and has also been supported by the Deutsche Forschungsgemeinschaft (DFG) in the Graduiertenkolleg GK 271 as well as by the European Community's Human Potential Programme under contract HPRN-CT-2000-00145. 


\begin{tabular}{rrrllll}
$L / a$ & $\beta=6 / g_{0}^{2}$ & $\kappa$ & $u=\bar{g}^{2}(L)$ & $Z_{\mathrm{P}}\left(g_{0}, L / a\right)$ & $Z_{\mathrm{P}}\left(g_{0}, 2 L / a\right)$ & $\Sigma_{\mathrm{P}}(u, a / L)$ \\
\hline & & & & & & \\
6 & 9.5000 & 0.1315322 & $0.9793(11)$ & $0.8265(4)$ & $0.7940(7)$ & $0.9606(10)$ \\
8 & 9.7341 & 0.1313050 & $0.9807(17)$ & $0.8195(5)$ & $0.7903(8)$ & $0.9643(11)$ \\
12 & 10.05755 & 0.1310691 & $0.9792(33)$ & $0.8095(5)$ & $0.7828(10)$ & $0.9670(14)$ \\
6 & 8.5000 & 0.1325094 & $1.1814(15)$ & $0.7985(5)$ & $0.7606(16)$ & $0.9526(21)$ \\
8 & 8.7223 & 0.1322907 & $1.1818(29)$ & $0.7896(9)$ & $0.7527(9)$ & $0.9533(14)$ \\
12 & 8.99366 & 0.1319754 & $1.1814(78)$ & $0.7793(5)$ & $0.7417(13)$ & $0.9517(19)$ \\
6 & 7.5000 & 0.1338150 & $1.5031(25)$ & $0.7565(4)$ & $0.7115(13)$ & $0.9405(18)$ \\
6 & 7.5420 & 0.1337050 & $1.5078(44)$ & $0.7604(4)$ & $0.7125(9)$ & $0.9370(13)$ \\
8 & 7.7206 & 0.1334970 & $1.5077(43)$ & $0.7486(8)$ & $0.7045(17)$ & $0.9411(25)$ \\
12 & 8.02599 & 0.1330633 & $1.503(12)$ & $0.7401(11)$ & $0.6967(12)$ & $0.9414(22)$ \\
6 & 6.6085 & 0.1352600 & $2.0146(56)$ & $0.7025(5)$ & $0.6406(7)$ & $0.9118(12)$ \\
8 & 6.8217 & 0.1348910 & $2.014(10)$ & $0.6921(11)$ & $0.6366(10)$ & $0.9198(21)$ \\
12 & 7.09300 & 0.1344320 & $2.014(20)$ & $0.6827(10)$ & $0.6236(15)$ & $0.9134(27)$ \\
6 & 6.1330 & 0.1361100 & $2.488(11)$ & $0.6584(7)$ & $0.5824(18)$ & $0.8845(29)$ \\
8 & 6.3229 & 0.1357673 & $2.479(13)$ & $0.6500(7)$ & $0.5755(21)$ & $0.8854(33)$ \\
12 & 6.63164 & 0.1352270 & $2.479(25)$ & $0.6431(10)$ & $0.5716(18)$ & $0.8888(33)$ \\
6 & 5.6215 & 0.1366650 & $3.326(20)$ & $0.5857(12)$ & $0.4898(25)$ & $0.8363(47)$ \\
8 & 5.8097 & 0.1366077 & $3.334(19)$ & $0.5810(12)$ & $0.4864(24)$ & $0.8371(45)$ \\
12 & 6.11816 & 0.1361387 & $3.334(49)$ & $0.5817(14)$ & $0.4888(28)$ & $0.8403(55)$ \\
\hline
\end{tabular}

Table 7: Results for the step scaling function $\Sigma_{\mathrm{P}}$.

\section{A Simulation results for $Z_{\mathrm{P}}$}

In Table 7 we collect the bare parameters and results of our simulations to compute $Z_{\mathrm{P}}$. Simulations on $L / a$ and $2 L / a$ lattices are required to extract the step scaling function $\Sigma_{\mathrm{P}}$ Eq. (3.1). At the three lowest couplings $\bar{g}^{2}(L)$ simulations have been performed using the 1-loop value of $c_{\mathrm{t}}$ [29], except for $L / a=6, \beta=7.5420$ and $L / a=8, \beta=7.7206$. For the latter parameters and the larger couplings the

\footnotetext{
${ }^{4}$ Note that the use of lowest order chiral perturbation theory in our work is not likely to be a significant source of difference to the other computations, since they do not report large deviations from lowest order chiral perturbation theory, see for example Ref. [3].
} 


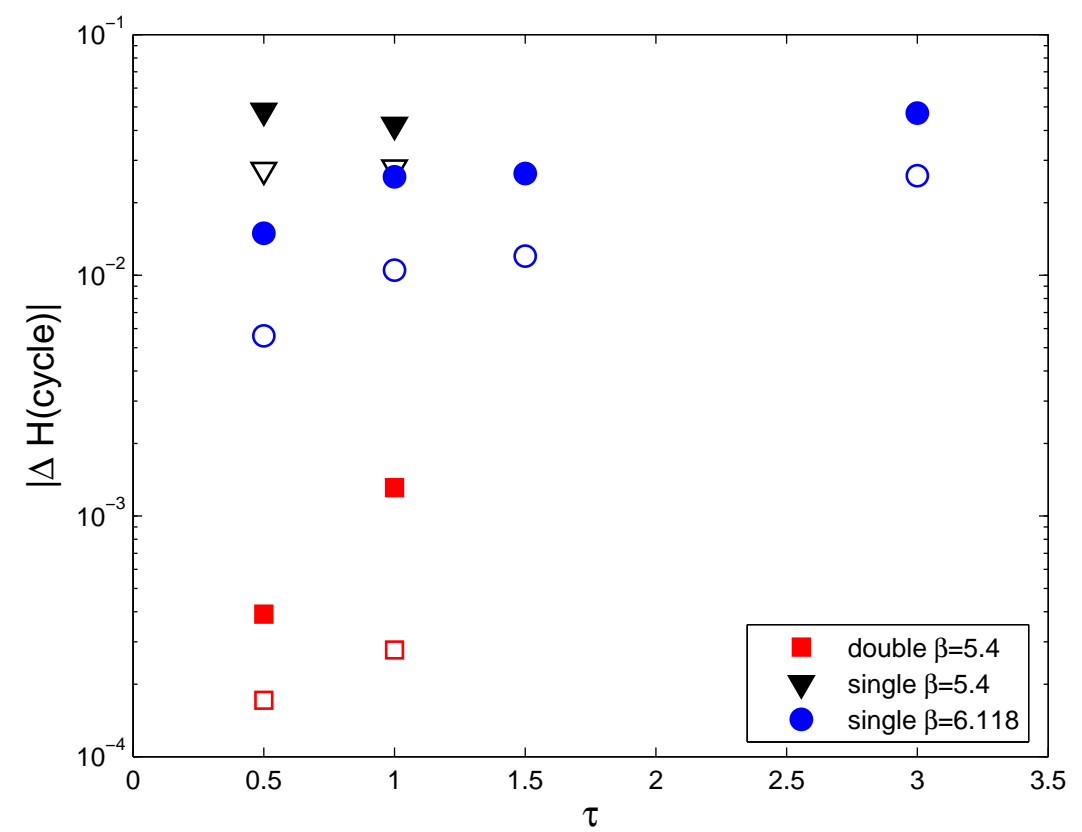

Figure 6: Reversibility violations in the HMC as a function of the trajectory length $\tau$ used in the reversibility tests. Empty symbols represent the average and filled symbols the maximal value of the Hamiltonian violation $\mid \Delta H$ (cycle) $\mid[11]$.

2-loop value of $c_{\mathrm{t}}$ [37] has been used. At the third lowest coupling $u \approx 1.5$ we checked at $L / a=6$ that there is no significant difference in $\Sigma_{\mathrm{P}}$ using the 1 - or 2-loop value for $c_{\mathrm{t}}$, as it is shown in Fig. 2.

To the statistical error of $\Sigma_{\mathrm{P}}$ we added in quadrature the error due to the uncertainty in the coupling $u$. The latter was estimated using the 1 -loop result $-\ln (2) d_{0}$ for the derivative of $\Sigma_{\mathrm{P}}$ with respect to $u$. This correction is tiny, it increases the errors of $\Sigma_{\mathrm{P}}$ by at most $5 \%$ at the largest coupling and lattice.

\section{B About the algorithm}

Our simulations have been performed using the Hybrid Monte Carlo algorithm with two pseudo-fermion fields as proposed by M. Hasenbusch $[11,55,56]$. Here we extend the study on algorithmic precision presented in Ref. [11].

For our largest lattice volume $L / a=24$ at $\beta=5.4$ we investigated the issue of reversibility. We looked at the quantity $\mid \Delta H$ (cycle) $\mid$ which measures the difference of the Hamiltonian for a cyclic trajectory [11]. In Fig. 6 we present data for the average $\mid \Delta H$ (cycle) $\mid$ (empty symbols) and the maximum of $\mid \Delta H$ (cycle) $\mid$ (filled symbols) on three sets of configurations. Sticking to single precision arithmetic 
we found for a trajectory length $\tau=0.5$ (that we use in the production run) an average value for $\mid \Delta H$ (cycle) $\mid$ (black triangles) that is larger by almost a factor five with respect to the simulations at $\beta=6.118$ reported in Ref. [11] (blue circles). We therefore repeated the simulation at $\beta=5.4$ in double precision arithmetic. At the same time we reduced the parameter $\epsilon^{2}$, defined ${ }^{5}$ as the requested accuracy in the conjugate gradient iteration, from $\epsilon^{2}=10^{-11}$ (as used with single precision) to $\epsilon^{2}=10^{-13}$. The results for double precision are the red squares in Fig. 6 . By comparing single with double precision, both the average and the maximal value of $\mid \Delta H$ (cycle) $\mid$ decrease by two orders of magnitude for double precision and these values are well below the values observed in Ref. [11]. Our statistics for the reversibility checks consists of 18 configurations analyzed at $\beta=5.4$ (both for single and double precision) and of 30 configurations analyzed at $\beta=6.118$. On a subset of the $\beta=5.4$ configurations we checked that within single precision by reducing $\epsilon^{2}$ to $10^{-13}$ (which one would expect to be the lower bound in single precision) the roundoff error in the Hamiltonian computation and consequently the value of $\mid \Delta H$ (cycle) $\mid$ remain unchanged.

Finally, we emphasize that we found no significant difference in the observables we computed at $\beta=5.4$ between the single and double precision simulations. The number quoted in Table 5 for $m_{\text {ref }}$ is from the simulation in double precision.

\section{References}

[1] S. Weinberg, Phys. Rev. D8 (1973) 3497.

[2] CP-PACS, A. Ali Khan et al., Phys. Rev. D65 (2002) 054505, heplat/0105015.

[3] JLQCD, S. Aoki et al., Phys. Rev. D68 (2003) 054502, hep-lat/0212039.

[4] QCDSF, M. Göckeler et al., (2004), hep-ph/0409312.

[5] CP-PACS, T. Ishikawa et al., Nucl. Phys. Proc. Suppl. 140 (2005) 225, heplat/0409124.

[6] HPQCD, C. Aubin et al., Phys. Rev. D70 (2004) 031504, hep-lat/0405022.

[7] ALPHA, S. Capitani, M. Lüscher, R. Sommer and H. Wittig, Nucl. Phys. B544 (1999) 669, hep-lat/9810063.

\footnotetext{
${ }^{5}$ We adopt here the commonly used convention to define $\epsilon^{2}$ as the square of the ratio between the norm of the residue vector and the norm of the source vector. It differs from the definition used in [11], where $\epsilon^{2}$ was normalized by the norm of the solution vector.
} 
[8] ALPHA, M. Della Morte et al., Nucl. Phys. B713 (2005) 378, heplat/0411025.

[9] ALPHA, F. Knechtli et al., Nucl. Phys. Proc. Suppl. 119 (2003) 320, heplat/0209025.

[10] ALPHA, F. Knechtli et al., Nucl. Phys. Proc. Suppl. 129 (2004) 814, heplat/0309074.

[11] ALPHA, M. Della Morte et al., Comput. Phys. Commun. 156 (2003) 62, hep-lat/0307008.

[12] M. Della Morte, R. Hoffmann and R. Sommer, JHEP 03 (2005) 029, heplat/0503003.

[13] M. Della Morte, R. Hoffmann, F. Knechtli, R. Sommer and U. Wolff, JHEP 07 (2005) 007, hep-lat/0505026.

[14] ALPHA, J. Rolf and S. Sint, JHEP 12 (2002) 007, hep-ph/0209255.

[15] UKQCD, A. Dougall, C.M. Maynard and C. McNeile, Nucl. Phys. Proc. Suppl. 140 (2005) 428, hep-lat/0409089.

[16] ALPHA, J. Heitger and R. Sommer, JHEP 02 (2004) 022, hep-lat/0310035.

[17] ALPHA, J. Garden, J. Heitger, R. Sommer and H. Wittig, Nucl. Phys. B571 (2000) 237, hep-lat/9906013.

[18] J. Gasser and H. Leutwyler, Nucl. Phys. B250 (1985) 465.

[19] H. Leutwyler, Phys. Lett. B378 (1996) 313, hep-ph/9602366.

[20] G. Amoros, J. Bijnens and P. Talavera, Nucl. Phys. B602 (2001) 87, hep$\mathrm{ph} / 0101127$.

[21] H. Wittig, Nucl. Phys. Proc. Suppl. 119 (2003) 59, hep-lat/0210025.

[22] D.B. Kaplan and A.V. Manohar, Phys. Rev. Lett. 56 (1986) 2004.

[23] M. Gell-Mann, R.J. Oakes and B. Renner, Phys. Rev. 175 (1968) 2195.

[24] ALPHA, S. Sint and P. Weisz, Nucl. Phys. B545 (1999) 529, hep-lat/9808013.

[25] K. Jansen et al., Phys. Lett. B372 (1996) 275, hep-lat/9512009.

[26] M. Lüscher, (1998), hep-lat/9802029. 
[27] K. Symanzik, Nucl. Phys. B190 (1981) 1.

[28] M. Lüscher, Nucl. Phys. B254 (1985) 52.

[29] M. Lüscher, R. Narayanan, P. Weisz and U. Wolff, Nucl. Phys. B384 (1992) 168, hep-lat/9207009.

[30] S. Sint, Nucl. Phys. B421 (1994) 135, hep-lat/9312079.

[31] S. Sint, Nucl. Phys. B451 (1995) 416, hep-lat/9504005.

[32] S. Sint and R. Sommer, Nucl. Phys. B465 (1996) 71, hep-lat/9508012.

[33] M. Lüscher, S. Sint, R. Sommer and P. Weisz, Nucl. Phys. B478 (1996) 365, hep-lat/9605038.

[34] ALPHA, K. Jansen and R. Sommer, Nucl. Phys. B530 (1998) 185, heplat/9803017.

[35] S. Sint and P. Weisz, Nucl. Phys. B502 (1997) 251, hep-lat/9704001.

[36] ALPHA, M. Guagnelli et al., Nucl. Phys. B595 (2001) 44, hep-lat/0009021.

[37] AlPHA, A. Bode, P. Weisz and U. Wolff, Nucl. Phys. B576 (2000) 517, hep-lat/9911018.

[38] R. Sommer, Nucl. Phys. B411 (1994) 839, hep-lat/9310022.

[39] ALPHA, U. Wolff, Comput. Phys. Commun. 156 (2004) 143, heplat/0306017.

[40] ALPHA, R. Sommer et al., Nucl. Phys. Proc. Suppl. 129 (2004) 405, heplat/0309171.

[41] ALPHA, M. Della Morte, R. Hoffmann, F. Knechtli and U. Wolff, Comput. Phys. Commun. 165 (2005) 49, hep-lat/0405017.

[42] J. Gasser and H. Leutwyler, Phys. Rept. 87 (1982) 77.

[43] J. Gasser and H. Leutwyler, Ann. Phys. 158 (1984) 142.

[44] J. Bijnens, G. Ecker and J. Gasser, (1994), hep-ph/9411232.

[45] T. van Ritbergen, J.A.M. Vermaseren and S.A. Larin, Phys. Lett. B400 (1997) 379, hep-ph/9701390. 
[46] K.G. Chetyrkin, Phys. Lett. B404 (1997) 161, hep-ph/9703278.

[47] J.A.M. Vermaseren, S.A. Larin and T. van Ritbergen, Phys. Lett. B405 (1997) 327, hep-ph/9703284.

[48] M. Czakon, Nucl. Phys. B710 (2005) 485, hep-ph/0411261.

[49] M. Lüscher, JHEP 05 (2003) 052, hep-lat/0304007.

[50] M. Lüscher, Comput. Phys. Commun. 165 (2005) 199, hep-lat/0409106.

[51] C. Urbach, K. Jansen, A. Shindler and U. Wenger, (2005), hep-lat/0506011.

[52] F. Bodin et al., Nucl. Phys. Proc. Suppl. 106 (2002) 173, hep-lat/0110197.

[53] ApeNEXT, F. Bodin et al., Nucl. Phys. Proc. Suppl. 140 (2005) 176.

[54] ALPHA, M. Guagnelli, J. Heitger, R. Sommer and H. Wittig, Nucl. Phys. B560 (1999) 465, hep-lat/9903040.

[55] M. Hasenbusch, Phys. Lett. B519 (2001) 177, hep-lat/0107019.

[56] M. Hasenbusch and K. Jansen, Nucl. Phys. B659 (2003) 299, heplat/0211042. 